\title{
How do we use high-frequency oscillation: Primary ventilation, rescue therapy or switch directly to early extracorporeal membrane oxygenation?
}

More than 40 years ago, the paediatric intensivist Charlie Bryan accidently discovered the concept of oscillation during laboratory experiments. ${ }^{[1]}$ In high-frequency oscillation (HFO), a piston generates high-speed, low-volume waves to enable gas exchange, while continuous gas flow maintains lung recruitment throughout the ventilation cycle. The patient is ventilated on the deflation limb of the pressure-volume curve where compliance is better and, theoretically, develops less ventilator-induced lung injury (VILI)..$^{[2]}$

The usefulness of HFO has been questioned following adult respiratory distress syndrome (ARDS) trials refuting the benefit of HFO above conventional mechanical ventilation (CMV). The large ARDS OSCILLATE $^{[3]}$ multicentre randomised control trial (RCT) compared early HFO to lung-protective CMV. The study was stopped early because of significantly lower in-hospital survival in the HFO group. Also, significantly more vasoactive, sedation and paralysis drugs were used in this group.

The ARDS OSCAR ${ }^{[4]}$ multicentre RCT showed improved oxygenation with HFO use compared with lung protective CMV, but no significant 30-day survival benefit. No significant difference in vasoactive drug use between the groups was found. Furthermore, the cost of treatment 1 year after the ARDS episode was significantly higher in the HFO group. ${ }^{[5]}$

A meta-analysis of seven adult ARDS RCTs compared HFO with $\mathrm{CMV}$, and did not confirm better 30-day mortality in patients who received HFO. HFO improved oxygenation significantly, but the patients needed longer ventilation. There was no difference in length of intensive care unit (ICU) stay, paralysis use, barotrauma or haemodynamic compromise between the groups. ${ }^{[6]}$

The only prospective multicentre RCT in children comparing HFO with CMV was conducted in the early 1990s. ${ }^{[7]}$ The HFO group needed significantly less supplemental oxygen at 30 days, and there was no difference in duration of ventilation, pneumothoraxes or mortality between the two groups. A large retrospective multicentre, observational paediatric ARDS (PARDS) study compared early HFO, late HFO and CMV. Both HFO arms showed an increase in length of ventilation, paediatric ICU (PICU) stay and mortality ${ }^{[8]}$ However, this retrospective study has been met with some scepticism due to concerns about bias, and controversial methods.

Despite the equivocal outcomes in adult trials and the paucity of paediatric data, for the past two decades HFO has remained a prominent rescue therapy in PICUs around the world. Yet clear consensus guidelines are still lacking. The difficulty with setting guidelines is related to the huge variability in pathophysiology, aetiology, lung maturity and size of PARDS patients. Expert opinion agrees on rescue HFO when CMV fails, and suggests extracorporeal membrane oxygenation (ECMO) when CMV and/or HFO fail in reversible disease ${ }^{[9]}$ ECMO achieves less VILI, clears carbon dioxide $\left(\mathrm{CO}_{2}\right)$ effectively and supports the cardiovascular system. However, it is not readily accessible in low- and middle-income countries.

In this issue, Cawood et al. ${ }^{[10]}$ describe a 2-year single-centre experience of rescue HFO in a South African (SA) PICU without
ECMO availability. The study confirms improved oxygenation when using HFO, in agreement with international evidence. The use of rescue HFO is slightly higher in this study than has been previously described, leaving one to speculate on the CMV strategies used prior to initiating rescue $\mathrm{HFO}$

The majority of publications on HFO describe its use for ARDS-related hypoxia. However, in this study, a large number of children (slightly less than half the cohort) needed rescue HFO for uncontrollable respiratory acidosis. The two extremes of hypoxic and hypercarbic respiratory failure in this population reflect the pathophysiological diversity of acute paediatric respiratory failure. The ability of HFO to uncouple ventilation and oxygenation makes HFO a valuable modality in the treatment armamentarium against all types of respiratory failure.

Pediatric Acute Lung Injury Consensus Conference (PALICC) guidelines recommend permissive hypercapnia, maintaining a $\mathrm{pH} 7.15$ $7.30 .^{[11]}$ In this study, children with refractory hypercarbia had a median $\mathrm{pH}$ of 6.92 prior to transitioning to HFO. This degree of acidosis has deleterious immunological, vascular and cardiac effects. It is therefore appropriate to escalate treatment, and Cawood et al. ${ }^{[10]}$ confirm the efficacy of $\mathrm{HFO}$ to clear $\mathrm{CO}_{2}$.

Furthermore, Cawood et al. ${ }^{[10]}$ describe more air leaks (11.8\%) than reported in the literature. Pressure-wave damping between the upper airways and alveoli depends on the homogeneity and pathophysiological process in the lung. Over-distension and barotrauma is a major risk in the unrecruited lung with inhomogeneous airspace disease. It is therefore important to ensure full lung recruitment before increasing HFO amplitude to generate higher tidal volumes in an attempt to control $\mathrm{CO}_{2}$. Dyssynchronous spontaneous breathing on $\mathrm{HFO}$ is a risk for pneumothoraxes. Sedation strategies were not discussed in this study, and may have influenced the number of air leaks. Lung compliance and airway resistance improve as the disease resolves, and aggressive weaning should be undertaken to prevent barotrauma.

The study also describes higher vasoactive requirements $(55.9 \%$ of the study population) than previously reported. Of note is the higher $(7 \mathrm{cmH} 2 \mathrm{O})$ median increase in mean airway pressure (MAP) from $\mathrm{CMV}$ to HFO than recommended, and may explain the cardiovascular instability. ${ }^{[11]}$ Experts advocate a gradual increase in MAP to open the lung.

Lastly, the mortality in this study was $22.7 \%$ higher than predicted by admission paediatric index of mortality 3 (PIM3) scores. In PARDS children, immune deficiency is associated with higher mortality but was not analysed. Oxygenation index at 24 hours after initiating HFO is the best predictor of mortality ${ }^{[12]}$ These parameters could have shed light on the higher-than-expected mortality, but were not included.

In summary, this first report on paediatric HFO use in SA fills a knowledge gap, and confirms the efficacy of HFO within the SA context. Furthermore, the work reports variability in practice, and confirms the need for clear guidelines on approach and on the timing of HFO. 


\section{B Rossouw, MD}

Division of Critical Care and Children's Heart Diseases, School of Child and Adolescent Health, Red Cross War Memorial Children's Hospital,

University of Cape Town

beyra.rossouw@uct.ac.za

South Afr J Crit Care 2019;35(2):41-42. https://doi.org/10.7196/SAJCC.2019.v35i2.415

1. Meyers M, Rodrigues N, Ari A. High-frequency oscillatory ventilation: A narrative review. Can J Respir Ther 2019;55:40-46. https://doi.org/ 10.29390/cjrt-2019-004

2. Kneyber MC, van Heerde M, Markhorst DG. Reflections on paediatric high-frequency oscillatory ventilation from a physiologic perspective. Respir Care 2012;57(9):1496-1504. https://doi.org/ $10.4187 /$ respcare. 01571

3. Ferguson ND, Cook DJ, Guyatt GH, Mehta S, Hand L, OSCILLATE Trial Investigators, Canadian Critical Care Trials Group. High-frequency oscillation in early acute respiratory distress syndrome. New Engl J Med 2013;368(9):795-785. https://doi.org/10.1056/NEJMoa1215554

4. Young D, Lamb SE, Shah S, OSCAR Study Group. High-frequency oscillation for acute respiratory distress syndrome. New Engl J Med 2013;368(9):806-813. https://doi.org/10.1056/ NEJMoa1215716
5. Lall R, Hamilton P, Young D, OSCAR collaborators. A randomised controlled trial and costeffectiveness analysis of high-frequency oscillatory ventilation against conventional artificial ventilation for adults with acute respiratory distress syndrome. The OSCAR (OSCillation in ARDS) study. Health Tech Assess 2015;19(23):1-177. https://doi.org/10.3310/hta19230

6. Maitra S, Bhattacharjee S, Khanna P, Baidya DK. High-frequency ventilation does not provide mortality benefit in comparison with conventional lung-protective ventilation in acute respiratory distress syndrome. Anesthesiology 2015;122(4):841-851. https://doi.org/10.1097/ ALN.0000000000000306

7. Arnold JH, Hanson JH, Toro-Figuero LO, Gutiérrez J, Berens RJ, Anglin DL. Prospective randomized comparison of high-frequency oscillatory ventilation and conventional mechanical ventilation in pediatric respiratory failure. Crit Care Med 1994;22(10):1530-1539

8. Gupta P, Green JW, Tang X, et al. Comparison of high-frequency oscillatory ventilation and conventional mechanical ventilation in pediatric respiratory failure. JAMA Pediat 2014;168(3):243-249. https://doi.org/10.1001/jamapediatrics.2013.4463

9. Kneyber MCJ, de Luca D, Calderini E, Section Respiratory Failure of the European Society for Paediatric and Neonatal Intensive Care. Recommendations for mechanical ventilation of critically ill children from the Paediatric Mechanical Ventilation Consensus Conference (PEMVECC). Intensive Care Med 2017;43(12):1764-1780. https://doi.org/ 10.1007/s00134-017-4920-z

10. Cawood S, Rae B, Naidoo KD. High-frequency oscillatory ventilation in a tertiary intensive care unit in an academic hospital in Johannesburg, South Africa. South Afr J Crit Care 2019;35(2):xxxx. https://doi.org/10.7196/SAJCC.2019.v35i2.370

11. Arnold JH. High-frequency ventilation in the pediatric intensive care unit. Pediatr Crit Care Med 2000;1(2):93-99.

12. Rettig JS, Smallwood CD, Walsh BK, et al. High-frequency oscillatory ventilation in pediatric acute lung injury: A multicentre international experience. Crit Care Med 2015;43(12):2660-2667. https://doi.org/ 10.1097/CCM.0000000000001278 